\title{
SUPERCONDUCTING CAVITIES FOR THE REDUCED-BETA SECTION OF A PROTON LINAC
}

\author{
C. Pasotti, P. Pittana and M. Svandrlik, \\ Sincrotrone Trieste, S. S. 14 per Basovizza, km 163.5, 34012 Trieste, Italy
}

Abstract

A proton linear accelerator with energies beyond 1.0 $\mathrm{GeV}$ has been proposed in [1] where the LEP2 superconducting ( SC ) cavities are to be used after decommissioning of LEP, in the high energy part of the linac. Energies ranging from $100 \mathrm{MeV}$ to $1.0 \mathrm{GeV}$ will be covered by superconducting cavities designed to accelerate protons with reduced value of beta. The basic idea is to keep the shape of the spherical LEP cavities for $\beta=1.0$ and to adjust their length, for $\beta<1.0$ in order to get an optimal acceleration for low energy protons. Three different values of $\beta$ allow to cover the required range with a reasonable acceleration efficiency. The design of superconducting cavities for $\beta=0.50,0.625$ and 0.80 is presented here. In particular the choice of an elliptic iris profile is discussed.

\section{CAVITY DESIGN}

All the LEP2 radiofrequency equipment, such as the RF plants and HOM couplers could be used in a lower energy range, i.e. $100 \mathrm{MeV}$ up to $1 \mathrm{GeV}$, if new SC accelerating cavities with the same resonant frequency of $352 \mathrm{MHz}$ can accelerate the beam with an acceptable efficiency. Three reduced- $\beta$ SC cavities have to be designed with axial length $\mathrm{L}_{\text {cell }}=\beta \lambda / 2$.

A simple linear scaling [2] of the already existing LEP2 cavity [3] has shown a remarkable deterioration of the cavity electromagnetic (EM) parameters with respect to the LEP2 due to the smaller volume and the electric field density decrease along the cavity axis. For the cavity with $\beta=0.5$ the ratio between the peak surface electric field and the accelerating electric field $\mathrm{E}_{\mathrm{Sp}} / \mathrm{E}_{\mathrm{a}}$ is doubled with respect to LEP2. This ratio is one of the most important parameter for the $\mathrm{SC} \mathrm{Nb} / \mathrm{Cu}$ cavity since it is related to the field emission limitation and to the maximum gradient that the cavity can substain. An improvement of the cavity shape is then required.

The cavity profile should be optimized according to fixed design constraints coming from the SC design technique and the manifacturing experience. The doorbell geometry of the LEP2 cavities should be maintained to minimize the multipacting risk. In order to utilize the CERN equipment the external cavity diameter should be close to that of LEP2 cavities. Flat cell side walls should be avoided for mechanical stability reasons, their inclination should be $\geq 8^{\circ}-10^{\circ}$. The cell to cell coupling factor $\mathrm{K}$ should be kept sufficiently high to achieve acceptable field flatness, so the iris beam tube radius can not be decreased too much. The Higher Order Modes should not be trapped in the cavity, and this also limits the size of the beam tube radius.

All the simulations have been performed with the computer code OSCAR2D [4] and the EM cavity parameters have been calculated for normal conducting copper at room temperature.

\subsection{The reduced-beta cell with an Elliptic Iris Shape}

The design of the lowest $\beta=0.5$ cavity is the more troublesome to perform. This cavity is the smallest one with an axial length half of the LEP2 cavity.

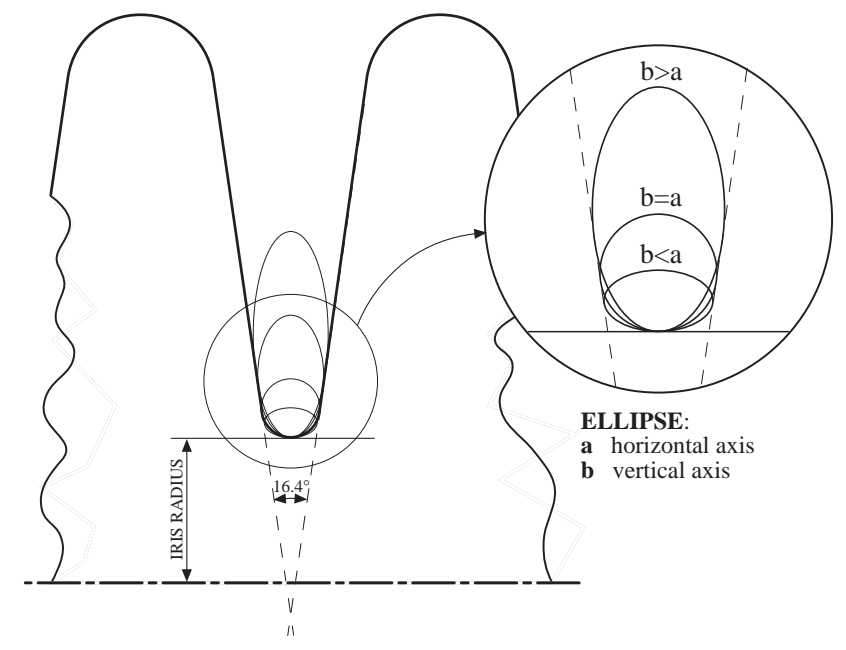

Figure 1: Sketch showing the elliptical iris shape. When the eccentricity is changed, the tangency condition to the side wall should be matched. The inclination of the side walls is kept constant.

A cavity with circular iris shape tailored for particles with $\beta=0.48$ has been presented in [5] and [6]. Starting from these shapes and taking into account the design specifications, the optimization of two cavity size parameters, the beam tube radius and the iris shape have been done to improve the EM cavity parameters. This optimization should take into account that these two parameters are strongly correlated. In fact, once the cavity side wall inclination has been fixed, the decreasing of the iris beam tube radius leads to the decreasing of the circular iris radius tangent to the side wall, as shown in figure 1 . The basic idea is to replace the circular iris radius with an elliptic shape and to investigate the behavior of main EM cavity parameters 
as a function of the elliptic sizes. It should be noted that the design guidelines do not allow to modify other cavity dimensions. In addition, when the beam iris radius is changed some adjustments of the cell diameter are required to tune the resonant frequency.

The variation of the $E_{s p}$ as a function of the ellipse eccentricity b/a is shown in figure 2 for different beam tube radii.

\section{$\operatorname{Esp}(\operatorname{Ea}=1.0 \mathrm{MV} / \mathrm{m})$}
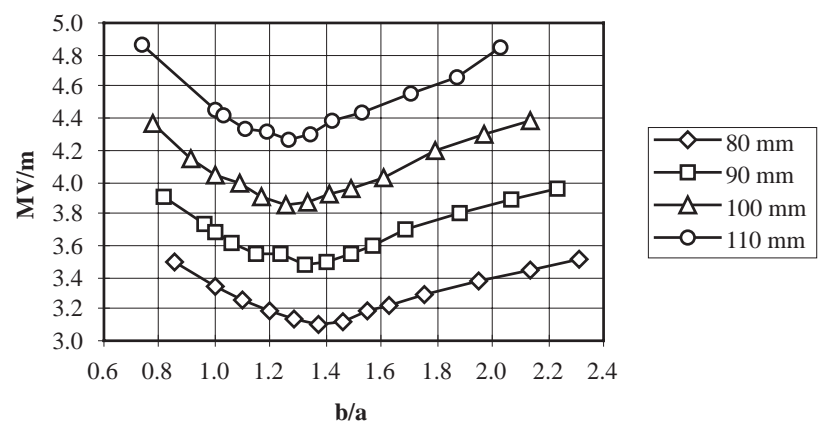

Figure 2: The $\mathrm{E}_{\mathrm{sp}}$ as a function of b/a.

The results show that the minimum values occur far from the circular profile. Lessening the beam tube radius the $\mathrm{E}_{\mathrm{sp}}$ becomes smaller.

The $E_{s p}$ value as a function of the ellipse's vertical axis $b$ is skctched in figure 3 for different beam tube radii.

$\operatorname{Esp}(\operatorname{Ea}=1.0 \mathrm{MV} / \mathrm{m})$

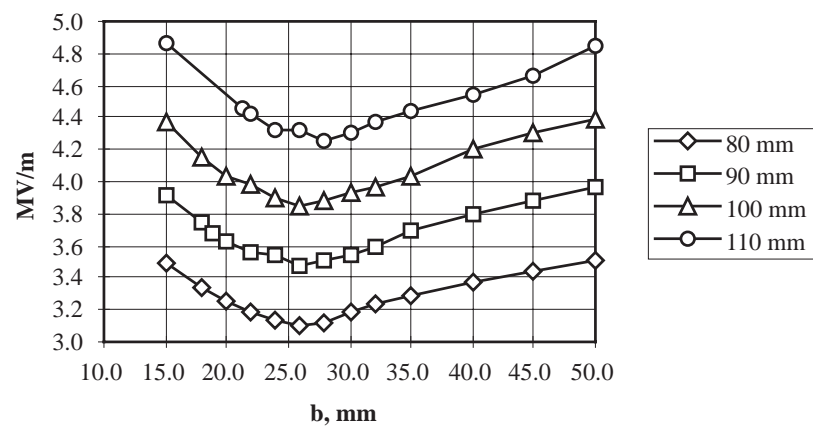

Figure 3: The $\mathrm{E}_{\mathrm{sp}}$ as a function of $\mathrm{b}$.

The requested reduction of the $\mathrm{E}_{\mathrm{sp}}$ with respect to the circular iris profile ranges from $-4.6 \%(\mathrm{r}=100 \mathrm{~mm})$ to $-7.2 \%$ ( $r=80 \mathrm{~mm}$ ). The other EM cavity parameters are not influenced by this elliptic profile. The elliptical shape of the iris seems to be a good alternative that matches the SC design specifications.

\subsection{The EA-1 cell design for $\beta=0.5$}

The present choice of $\beta=0.5$ for the first accelerating structure allows a more comfortable design, since the cavity axial length is increased. All the previous results on the elliptical iris profile still hold.
Due to the increased sizes, another cavity shape parameter can be further optimized, the hat radius $\mathrm{R}$. Preliminary studies suggest to keep $R \geq 50 \mathrm{~mm}$. Once the elliptical iris profile is optimized the $E_{s p}$ value for both hat radius $\mathrm{R}=55 \mathrm{~mm}$ and $\mathrm{R}=50 \mathrm{~mm}$ does not change. But a big difference is found in the shunt impedance value, which is $30 \%$ greater for $\mathrm{R}=55 \mathrm{~mm}$. The choice of which profile is better depends on the possibility to use the existing CERN manifacturing tools or on the possibility to save the $30 \%$ of the refrigerator power. Figure 4 shows one of the optimized profile and table 1 the $\mathrm{TM}_{010} \pi$-mode for both the shapes.

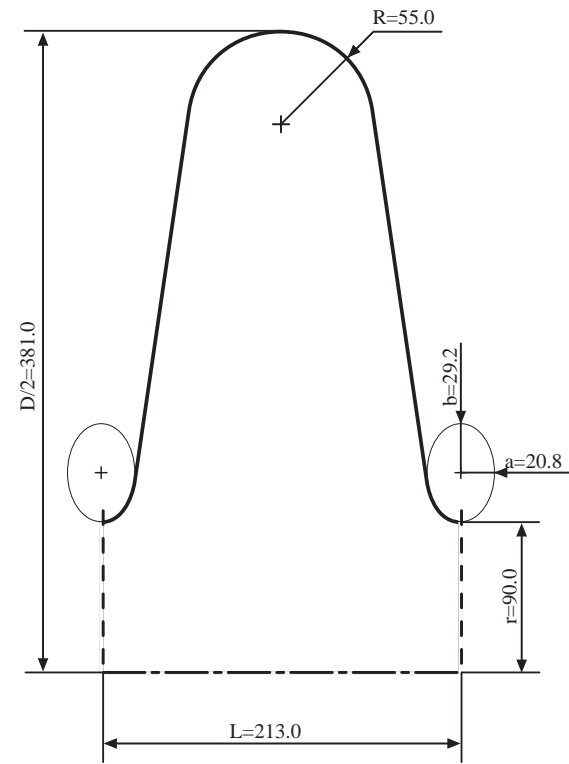

Figure 4: The EA-1 cell final shape.

\begin{tabular}{|l|l|l|l|}
\hline & $\mathrm{R}=55 \mathrm{~mm}$ & $\mathrm{R}=50 \mathrm{~mm}$ & LEP2 \\
\hline $\mathrm{Fr}, \mathrm{MHz}$ & 352.2 & 352.2 & 352.0 \\
$\mathrm{~K}, \%$ & 1.55 & 1.92 & 1.76 \\
$\mathrm{E}_{\mathrm{sp}} / \mathrm{E}_{\mathrm{a}}$ & 3.29 & 3.33 & 2.35 \\
$\mathrm{H}_{\mathrm{sp}} / \mathrm{E}_{\mathrm{a}}$ Gauss/MV/m & 62.4 & 71.2 & 39.1 \\
$\mathrm{Z}, \mathrm{M} \Omega$ & 0.47 & 0.37 & 3.51 \\
$\mathrm{Q}$ & 29400 & 27800 & 57600 \\
\hline
\end{tabular}

Table 1: Parameters of the $\mathrm{TM}_{010} \pi$-mode for both the two EA-1 cells and the LEP2 cell, as a reference values.

\subsection{The EA2 cell design for $\beta=0.625$}

The increase of the axial cell length also enlarge the hat radius.

It has been shown that the $\mathrm{E}_{\mathrm{sp}}$ does not change once the elliptical profile has been chosen. This result allows to start the design with a cell shape that matches the requested specifications and then to optimize the elliptical iris profile.

To keep the $\mathrm{K}$ value greater than $1.5 \%$, the hat radius for the EA-2 cell should be greater than $70 \mathrm{~mm}$. Better values of the peak surface magnetic field and shunt impedance are found for $\mathrm{R}=80 \mathrm{~mm}$ and beam tube 
radius $r=100 \mathrm{~mm}$. Starting with these dimensions the elliptic iris shape is optimized. The minimum value of $\mathrm{E}_{\mathrm{sp}}$ is reached for an ellipsis axis $\mathrm{b}=48 \mathrm{~mm}$ and $\mathrm{b} / \mathrm{a}=1.59$. Table 2 shows the cavity EM parameters.

\subsection{The EA3 cell design for $\beta=0.80$}

This is the last and less difficult cavity to design, since, due to its axial length, the hat radius $\mathrm{R}$ ranges from 51.0 $\mathrm{mm}$ to $65.0 \mathrm{~mm}$.

Trying to increase the shunt impedance with a larger value of $R$ and smaller value of $r$ leads to an increase of the peak surface electric field. A good compromise is reached with $\mathrm{R}=120 \mathrm{~mm}$ and $\mathrm{r}=110 \mathrm{~mm}$. The miminum value of $E_{\mathrm{sp}}$ is found as before, for an elliptic axis $\mathrm{b}=58-60 \mathrm{~mm}$ and an eccentricity $\mathrm{b} / \mathrm{a}=1.6$.

\begin{tabular}{|l|l|l|l|}
\hline & EA-2 & EA-3 & LEP2 \\
\hline Fr MHz & 352.2 & 352.2 & 352.0 \\
$\mathrm{~K} \%$ & 1.57 & 1.58 & 1.76 \\
$\mathrm{E}_{\mathrm{sp}} / \mathrm{E}_{\mathrm{a}}$ & 2.65 & 2.27 & 2.35 \\
$\mathrm{H}_{\mathrm{sp}} / \mathrm{E}_{\mathrm{a}}$ Gauss/MV/m & 54.1 & 45.2 & 39.1 \\
$\mathrm{Z}, \mathrm{M} \Omega$ & 0.94 & 1.95 & 3.51 \\
$\mathrm{Q}$ & 36900 & 47000 & 57600 \\
\hline
\end{tabular}

Table 2: EA-2 and EA-3 optimized cavity parameters.

\section{MULTICELL DESIGN}

The full cavity is made up of four cell structure. This number seems to be a good compromise between a cavity with a larger number of cells, which has more fabrication drawbacks and a smaller acceptance range in $\beta$, and a cavity with fewer cells, which has lower accelerating voltage for similar costs.

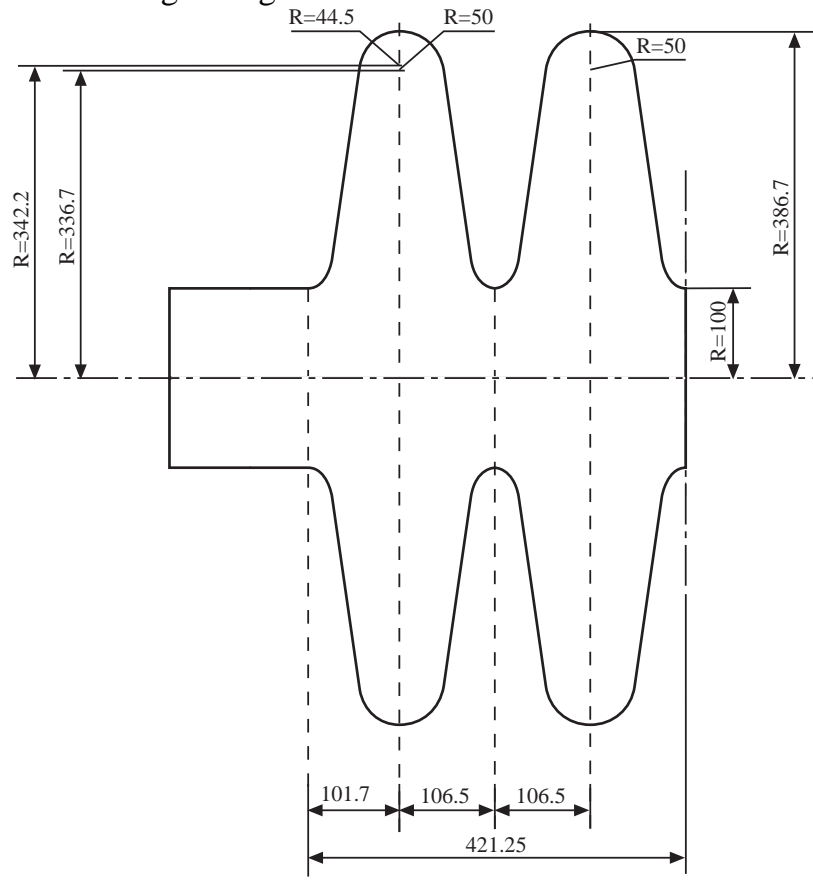

Figure 5: Half of theEA-1 cavity.
In all cavities the external cell has been adjusted to get an acceptable field flatness for the operating $\mathrm{TM}_{010}$ $\pi$-mode. To compensate this mode the cell hat radius of the external half-cell has been changed. The side wall inclination and the length of the axes of the ellipse have been kept constant by adjusting correspondingly the axial length of the external half-cell. In this way the peak electric field behaviour in the external cell iris region is similar to the one found for the single cell. The beam tube radius has been left untouched as well. Figure 5 shows the final result for the EA-1 cavity. Similar considerations hold for the EA-2 and EA-3 multicell design.

The acceleration efficiency $\eta$ of each cavity for all proton energies in consideration has been evaluated. Due to the tail behavior of the end cell electric field the $\eta$ reaches the peak value for a particle speed that is slightly higher than the $\beta=\beta_{0}$ value.

\begin{tabular}{|l|l|l|l|}
\hline Cavity & EA-1 & EA-2 & EA-3 \\
\hline Input Energy, MeV & 100 & 209 & 411 \\
$\eta$ at Input Energy & 0.64 & 0.86 & 0.83 \\
Output Energy, MeV & 209 & 411 & 997 \\
$\eta$ at Output Energy & 1.09 & 1.04 & 1.03 \\
Number of cavity & 32 & 36 & 80 \\
\hline
\end{tabular}

Table 3: Energy range, efficiency and number of cavities of the Linac sections. The $\eta$ normalization has been done for $\beta=\beta_{0}$.

\section{ACKNOWLEDGEMENTS}

The authors are pleased to acknowledge D. Boussard, E. Chiaveri and J. Tückmantel of CERN-SL, Geneva, and R. Parodi of INFN, Genoa, for their helpful discussions.

\section{REFERENCES}

[1] C. Rubbia and J.A. Rubio, "A Tentative Programme Towards a Full Scale Energy Amplifier", CERN/LHC/96-11 (EET), 15th July 1996.

[2] C. Pasotti, P. Pittana, M. Svandrlik, "LEP SC Cavities for Particles with $\beta<1$ ", Sincrotrone Trieste internal note August 1996, unpublished.

[3] E. Haebel, P. Marchand and J. Tückmantel, "An Improved Superconducting Cavity Design for LEP", CERN/EF/RF 84-2, 2nd April 1984.

[4] P. Fernandes and R. Parodi, "LALAGE-A Computer Program to Calculate the TM01 modes of Cylindrically Symmetrical Multicell Resonant Structures", Particle Accelerators, 1982, Vol. 12 pp. 131-137.

[5] J. Tückmantel, "Superconducting Cavities for Reduced $\beta "$, CERN-SL-Note 96-64 RF, 1996 Geneva.

[6] C. Pasotti, P. Pittana and M. Svandrlik, "LEP SC cavities modified for Particles with $\beta<1$ ", minutes of the Meeting on EA-Accelerator held at CERN, Geneva, on the 23rd September 1996. 